Zusammenfassung.

Wir haben den ganzen Koagulationsworgang der kolloiden Goldlösung unter der graduellen Einwirkung einiger Elektrolyte untersucht, indem wir von dem Momente ausgingen, wo die Koagulation sich noch nicht durch Farbenänderung bemerkbar machte, bis zu dem Momente, wo die mit Elektrolvten versetze Goldlösung ganz entfärbe wird, sobald die disperse Phase gänzlich sedimentiert ist.

1. Auf dem Wege ultranikroskopischer Beobachtungen über die Einwirkung anwachsender Mengen von Blektrolyten während einer bestimmten Zeit und gleichfalls auch auf dem Wege ultramikroskopischer Untersuchungen über die Kongulationsgeschwindigkeit der mit einer bestimmten Menge von Elektrolyten versetzien Goldlosung haben wir erwiesen, dab:

a) in der ultramikroskopischen Struktur des Goldhydrosols, weichem kleine Mengen von Elektrolyten $\left(0,5 \cdot 10^{-3}\right.$ bis $3,5 \cdot 10^{-3}$ Millimol pro $10 \mathrm{ccm}$ des Hydrosols) zugeselzt sind, welches aber trotzdem längere Zeit hindurch (ca. 1 Woche) seine ursprüngliche rote Farbe erhält, einige Aenderungen vor sich gehen: die Zahl seiner Submikronen wächst ein wenig auf Kosten der Amikronen (Tabelle II, letzte Spalte);

b) die Farbenänderungen des Goldhydrosols, welche durch die Einwirkung von Elektrolyten entstehen, im Verein mit ultrarnikroskopischen Strukturänderungen auftreten, welche stufen- artig :n der Richtung der Zunahne der Gröbe und Abnahme der Anzahi der Goldteilchen verlaufen.

2. Die elektrokinetischen Messungen über die graduellen Koagulationswirkungen einiger Elekrrolyte $\left(\mathrm{SrCl}_{3}, \mathrm{CaCl}_{2}, \mathrm{MgCl}_{2}, \mathrm{LiCl}, \mathrm{RbCl}\right)$ fúbren $z u$ folgender Resultaten:

a) in Uebereinstimmung mit den Ergebnissen von fritheren Forschungen (W.R.W hit ne y und J. C. Blake, E. F. Burton a. a. l. c.) entiaden die Elektrolyte die disperse Phase allmählich und laden sie schlieblich um;

b) die Elektrolyte, welche gleichwertige Kationen (sowohl die einwertigen $\mathrm{Li}$, $\mathrm{Rb}$, als auch zweiwertige $\mathrm{Sr}^{*}, \mathrm{Ca}^{*}, \mathrm{Mg}^{*}$ ) und identische Anionen $\left(\mathrm{Cl}^{\prime}\right)$ enthalten, koagulieren das Goldhydrosol, wie man schon bei ultramikroskopischen Untersuchungen beobachtet, in ungieichem Maße. Es ist wahrscheinlich, dab in unserem Falle auf die Koagulationsfăhigkeit von Elektrolyten auf die kolloide Goldlösung ihr elektrolytischer Dissoziationsgrad von Einflub ist.

Nehenher wurden noch weitere Beobachtungen gemacht, besonders über die elektrischen Eigenschaften des Goldhydrosols. Diese Beobachtungen gedenke ich in dieser Zeitschrift zu veroffentlichen, sobald ich alle noch dazu gebörigen Versuche soweit beendigt habe, daß ich die Resuitate meiner bisherigen Forschungen ergänzen und verallgemeinern kann.

\title{
Ueber den Einfluß der fettlöslichen Stoffe auf die Viskosität und die Oberflächenspannung des Olivenöls.
}

Von Franz Buban ovic (Stockholm). (Elagegangen am 19. Januar 1912)

(Aus den physiologischen Laboratorium der Reichsuniversität in Groningen)

Bei den Untersuchungen über die Phagozytose der Pferdeblutleukozyten unter dern Finflub der fettloslichen Substanzen ) wurde die interessante Tatsache gefunden, dab die untersuchten Stoffe das phagozyläre Vermögen der weiben Blutkörperchen im ansehnlichen Mabe beschleunigen. So z. B. steigert $\mathrm{CHCl}_{3}$ in einer Verdünnung von 1:100000 das phagozytäre Veımögen der Phagozyten von 41,2 Proz auf 60,1 Proz.

Als man nach der Ursache dieser sowohi für Physiologie, wie auch für Pathologie wichtigen

1) H.J. Ha mburger, J. de Ha a n und F. Bubanovid, Konikl. Akad. van Wetensch. Ansterdam, Wisk. en Naik. Afd. 19, 894-914 (1911). Siehe auch Arch. néerland. scient. exat. et nat. (2. Serie) 1.124 (1911).
Erscheinung suchte, lag es nahe, zu denken, daf der physikalische Zustand (die Viskosität und die Oberilachenspannung) der sogenannten Lipoidmembran der Phagozyten durch das Auflösen von sehr kleinen Quantitäten der fettlöslichen Stoffe in dera Sinne verăndert wird, daB eine schnellere Bewegung der Phagozyten und so auch ein größeres phagozytäres Vermögen mögilich gemacht wird.

Auf die Veranlassung meines hochgeschatzten Lehrers Prof. H. J. H a mburger entschlob ich mich, dieser Frage nüher zu treten.

Nachden ich vergebens aus Rindergehirn eine fur las Studium der Veränderungen der Viskositât und derOberfiächenspannung geeignete Lipoidsubstanz za isolieren versuchte (alle besitzen 
einen zu hohen Schmelzpunkt), nahm ich einfach als Versuchsmaterial zu diesem Zwecke das Olivenöl, eine bei vielen analogen Untersuchungen als lipoidathnlich benutzte Substariz.

Ich legte mir dann die frage vor, ob die genannten physikalischen Konstanten des Olivenöls unter dem Einfluk von sehr kleinen Quantităten der fettlöslichen Substanzen eine merkliche und bedeutende Veränderung erfahren.

\section{A. Die Veränderung der Viskosität.}

Diese wurde auf die folgende Weise studiert.

Es handelt sich darum, ob schon Spuren von einer fettlöslichen Substanz dem Olivenol beigefügt seine Viskositätskonstante ändern. Deswegen sollte ein Viskosimeter von hoher Empfindlichkeit und Genauigkeit benutzt werden. Ein solcher Apparat wurde mir durch die Gùte des Herm Dozenten A. W. Vis s e r (Groningen) zurVerfügung gestellt. Er hat nämlich vor kurzer Zeit einen neuen Viskosimeter nach dem Prinzip Ha ro-Determann konstruiert, dessen Genauigkeit bis zur dritten Dezimale des Viskositätskoeffizienten geht $^{2}$ ).

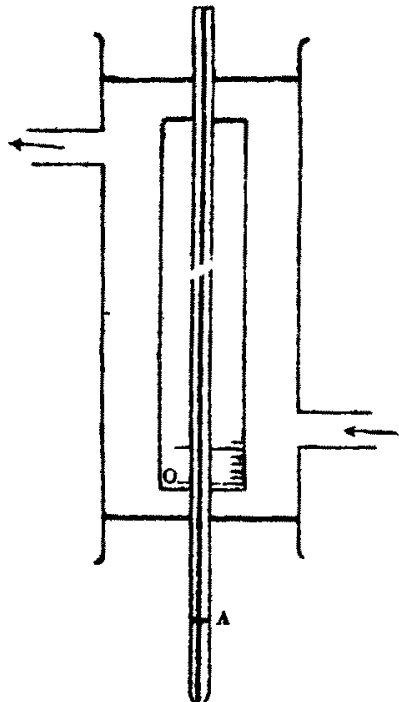

Fig. 1
Der Apparat ist in Fig. 1 abgebildet. Er besteht aus einer $70 \mathrm{~cm}$ langen Kapillare von $\pm 0,5 \mathrm{~mm}$ Durchschnitt. Die beiden Enden sind in Punkte abgeschliffen. Am Kapillarrohr sind fünf Teilstriche angebracht, und zwar so, daB $\mathrm{AB}=\mathrm{CD}=\mathrm{EF}$ $=20 \mathrm{~cm}$ und $\mathrm{BC}$ $\Rightarrow \mathrm{DE}=5 \mathrm{~cm}$ ist.

Um dieses Kapillarrohr befindet sich ein Glasmantel, geschlossen mit Gummikorken $K_{1}$ und $K_{2}$, durch welche die Enden der Kapillare hinausragen. Mittels einer kupfernen Montur ist das Rohr drehbar gestellt auf zwei Kolonnen, welche durch Schrauben auf einer eisernen FuBplatte befestigt sind. Auf dieser Platte befinden sich auch die nôtigen Einrichtungen, mit welchen

2) M.Sijpkens, Viscositeit en viscosimetrie van het bloed. Diss. (Groningen 1911), 94123.

Der Apparat ist von Herrn J. J. Boo m, Mechaniker des physiologischen Laboratoriums in Groningen verfertigt und kann bei ihm bestellt werder. man das Kohr genau vertikal aufstellen kann. Der Glasmantel hat in der Mitte eine Seiten offnung, geschlossen mit einem dreimal durchbohrten Gummikork. Durch die mittelste Oeffnung des Korkes geht ein bis auf $1 / 10^{\circ} \mathrm{C}$ genaues Thermometer und durch die beiden anderen Oeffnungen der $\mathrm{Zu}$ - und Abflubgummischlauch, womit aus einem Thermostat mittels einer elektrischen Pumpe das Wasser von bekannter Temperatur um die Kapillare strömt.

Die Messunger sind folgendermaben ausgeführt.

Nachdem die Temperatur längere Zeit im Glasmantel auf $37^{\circ} \mathrm{C}$ konstant gehalten bei dieser Temperatur machte man die $\mathrm{Be}$ stimmungen, weil auch die Phagozytose bei $37^{\circ} \mathrm{C}$ immer untersucht war -.- wurde die ganz reine und trockene Kapillare mittels eines bei $A$ angesetzten Gummischlauches mit Olivenöl vollgesaugt. Nach Entfernung des Gummischlauches lieb man das Oel langsam bei $F$ ausflieben; man beförderte dies und reinigte zugleich das Kapillarende mit einem Stücke gepreBten Filtrierpapiers. Je mehr sich der obere Meniskus der Oelkolonne dem $E$ năherte, um so mehr wurde das Rohr in eine horizontale Lage gedreht, und als der Meniskus gerade bei $E$ stand, wurde das Rohr um $180^{\circ}$ von der ursprünglichen l.age gedreht und wieder g nau vertikal gestellt. Jetzt wartete man mit einer Lupe und mit elnem auf $1 / 10$ Sekunde genauen Chronometer in der Hand bis der obere Meniskus der Oelkolonne bei $D$ anlangte und bestimmte so die Durchlauf $z$ eit des oberen Meniskus bis $C$.

Wenn man das Rohr nachher schnell um $180^{\circ}$ umgedreht hat. so kann man die Durchlaufzeit von $C$ bis $D$ wieder bestimmen und so mit derselben Oelkolonne die vorige $\mathrm{Be}$ stimmung kontrollieren. Dies kann man weiter 10 bis 20 mal wiederholen und aus den ethaltenen Zahlen den Mittelwert der Durchlaufzeit bestimmen. So wurde auch bei den vorliegenden Untersuchungen verfahren.

Außer für das Olivenöl wurden auf diese Weise auch die Durchlaufzeiten für Lösungen des Chloroforms, Aethylalkohols und Kampfers in Olivenöl erhalten. Dabei ist. noch zu bemerken, daß besonders darauf geachtet wurde, das nach jeder Bestimmung die Kapillare sorgfältig gereinigt und getrocknet wurde. (Mittels Durchsaugens von: Chloroform-Aether - Alkohol -. Wasser -- Aikohol - Aether getrockneter und gereinigter Luft.)

Aus den erhaltenen Durchlaufzeiten und aus den bei derselben Temperatu bestimmten spes. 
Gewichten des Olivenöls und der einzelnen Lösungen berechnete man den Viskositäts koeffizienten aus der Formel $\eta=$ Cst, wo bei dem gebrauchten Apparat: $\mathrm{C}=$ eine Konstante, welche aus gevau gemessenen und geeichten Größen der Kapillare resultiert und $=0,0035869$ ist $^{3}$ ), $s=$ das spez. Gewicht und $t=$ die Durchlaufzeit in Sekunden ist.

Die Resultate der Messungen sind in der nachfolgenden Tabelle zusammengestellt.

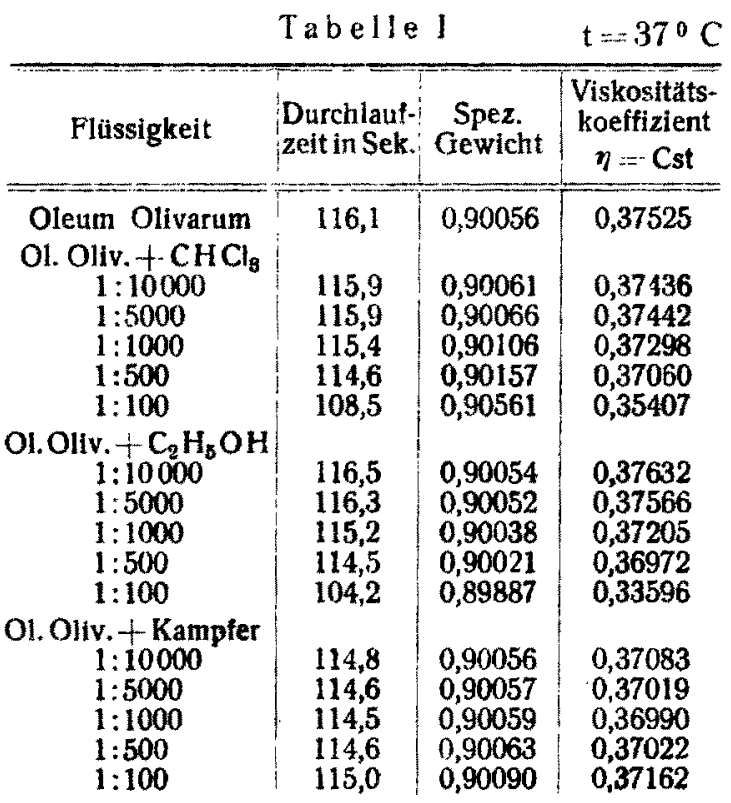

Aus den Daten dieser Tabelle geht hervor, daß kleine Quantitäten der fettlöslichen Stoffe, und zwar Chloroform, Aethylalkohol und $\mathrm{Kampfer}$ die Viskosität des Olivenöls $\mathrm{n} i \mathrm{ch} \mathrm{t}$ bedeutend und merklich beeinflussen.

\section{B. Die Verănderung der Oberflächen- spannung.}

Um den Einflußs von kleinen Quantitäten der fettlöslichen Substanzen auf diese physikalische Konstante des Olivenöls $\mathrm{zu}$ betrachten, wurde die Steighöhemethode gewählt. Die Messungen sind aus oben erwähntem Grunde auch hier bei $37^{\circ} \mathrm{C}$ ausgeführt.

$\mathrm{Zu}$ diesem $\mathrm{Zwecke}$ stellte sich die in Fig. 2 schematisch dargestellte Eirrichtung als zuverlässig beraus und zur Frhaltung der relativen und für komparative Vergleichungen nötigen Daten der Obertlächenspannung ausreichend.

Auf einer unten in einem Punkte abgeschliffenen Kapillare von $+0,25 \mathrm{~mm}$ Durch-

3) M. Sipkens, I.c. schnitt und $20 \mathrm{~cm}$ Länge wurde eine genaue Thermometerskala angebracht. Die Kapillare samt Skala wurde in einen Glasmantel mit zwei Gummikorken gebracht und das Ganze auf einem kupfernen Gestell so aufgestellt, dab die Kapillare fest in der vertikalen Lage hing. Durch die Oeffnungen des Mantels strömte aus einem Thermostat Wasser von $37^{\circ} \mathrm{C}$. Auf dem unteren, durch den Gummikork hinausragenden Teil der Kapillare war eine Marke $A$ angebracht.

Die Messungen wurden folgendermaßen ausgeführt:

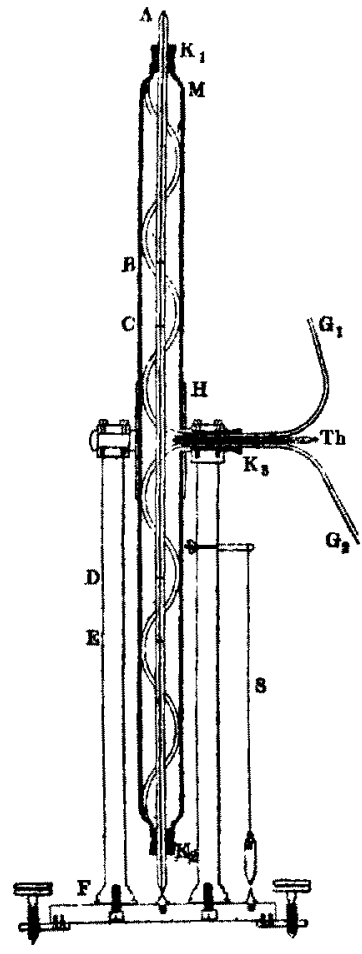

Fig. 2
Nachdem längere Zeit das Wasser von $37^{\circ} \mathrm{C}$ um die Kapillare zirkulierte und das Kapillarrohr nach bekannten Vorschriften tüchtig gereinigt und getrocknet war, wurde ein kleines Becherglas unter die untere Oeffnung der Kapillare gesetzt und mit Olivenöl oder einer anderen untersuchten Flüssigkeit so weit aufgefüllt, dah die Oberfläche der Plussigkeit gerade bis zur Marke $A$ reichte. Um nachher den Meniskus der Flüssigkeit in der Kapillare in Bewegung zu setzen, wurde von Zeit zu Zeit das Becherglas ein wenig auf und nieder geschoben. Als man die konstante Hohe der Flüssigkeit in der Kapillare erreichte, wurde der Stand des Meniskus mittels einer Lupe genau auf der Skala abgelesen.

Die Zahl der abgelesenen Millimeter drückte aber nicht die ganze Länge der Plüssigkeitssäule in der Kapillare aus, sondern nur von 0 der Skala ab. Weil aber der Abstand von $A$ bis 0 bei allen Messungen konstant blieb, so ergaben die abgelesenen Millimeter die r $\mathrm{l}$ a tiv e $\mathrm{n}$ Steighöhen, welche für die Untersuchung ausreichend waren.

Die Resultate betreffs der Lösungen des $\mathrm{Ch}$ lor of orms in Olivenol sind in der untenstehenden Tabelle angeführt. Mit Aethylalkohol 
und Kampfer wurden nur einige blinde Versuche gemacht.

\begin{tabular}{c|c}
\multicolumn{2}{c}{ Tabe lle II } \\
\hline Flussigkeit & $\begin{array}{c}t=37^{\circ} \mathrm{C} \\
\text { Relative Steighohe } \\
\text { in mm }\end{array}$ \\
\hline Chloroform & 3,5 \\
Oleum Olivarum & 19,0 \\
O1. Oliv. $+\mathrm{CHCl}_{8}$ & \\
$1: 10000$ & 18,9 \\
$1: 5000$ & 18,7 \\
$1: 1000$ & 18,8 \\
$1: 500$ & 18,7 \\
$1: 100$ & 18,6 \\
$1: 10$ & 17,3 \\
$1: 5$ & 14,5 \\
$1: 2$ & 11,8 \\
$1: 1$ & 10,3
\end{tabular}

Aus dieser Tabelle sieht man deutlich, daB kleine Quantităten des Chloroforms keinen merklichen Einfluß auf die Oberflächenspannung des Olivenöls ausüben. Einige blinde Versuche mit den Lösungen des Aethylalkohols und des Kampfers in Olivenöl lassen dasselbe für diese Substanzen vermuten.

\section{Zusammenfassung.}

1. Kleine Quantitäten der untersuchten fettloslichen Substanzen (Chloroform, Aethylalkohol und Kampfer) üben keinen merklichen Einfluß auf die Viskosităt und die Oberflächenspannung des Olivenöls aus.

2. Wenn man aus Versuchen mit Olivenöl schlieben darf, beeinflussen diese Substanzen in sehr kleinen Konzentrationen auch nicht die genannten physikalischen Konstanten der sogenannten Lipoidmembran der Phagozyten

(Aus dem physiologischen Laboratorium der Reichsuniversitat in Groningen.)

\title{
Ueber die Bildung der kolloiden Lösungen.
}

\author{
Von N. Pappadà) (Cremona) \\ (Eingegangen am 9. Februar 1912) \\ (Vorlăufige Mitteilung.)
}

In einigen meiner früheren Arbeiten habe ich behauptet, daB die Substanzen, welche die Suspension der kolloiden Teilchen bestimmen, keine chemisch indifferenten Körper für das Kolloid sind. Ich sagte, daB zwischen der Substanz und dem Kolloid eine chemische Reaktion stattfinden müsse, welche die Bildung leicht zersetzlicher, d. i. labiler Verbindungen zur Folge hat. Solche Verbindungen sind bei Gegenwart eines Ueberschusses des Kristalloides, welches die Lơsung begünstigt hat, beständig; sie werden aber unbeständig bei Gegenwart von viel Wasser. Die Eigenschaften jedes Kolloides und die wahrscheinliche chemische Affinităt zwischen Kolloid und Kristalloid, durch welche die Suspension bestimmt wird, bewogen mich zur Annahme einer chemischen Beziehung zwischen den beiden Körpern: Lösung und Gelöstes. Aus diesen Gründen habe ich systematische Versuche über die Bildung der Lösung von Kolloiden angestellt.

Der große Umfang dieser Arbeit, die vielfaltigen und verschiedenen Untersuchungen erlauben es mir nicht, alle experimentellen Ergebnisse qualitativer und quantitativer Art sofort zu publizieren, weshalb ich zum Zwecke, dieses weite Gebiet meiner Bearbeitung vor-

2) Uebersetzt von J. Matula, Wien. zub\&halten, in dieser vorläufigen Mitteilung einige Resultate von gröberem Inter esse mitteilen will; und zwar handelt es sich um die Herstellung eines positiven (Eisenhydroxyd) und eines negativen Kolloides (Berlinerblau).

Das Eisenchlorid hat die Eigenschaft nicht nur das Eisenhydroxyd, sondern auch andere Hydrate zu losen. Ich habe Lösungen von $\mathrm{Cr}(\mathrm{OH})_{3}$ und $\mathrm{Al}(\mathrm{OH})_{3}$ in ziemlich konzentriertem Eisenchlorid herstellen können. $\mathrm{Bi}(\mathrm{OH})_{3}$ lost sich nur in sehr konzentriertem Chlorid; durch Hinzufügung von Wasser wird es als gelbliches Pulver ausgefallt.

In Eisenchlorid von mittleren Konzentrationen lösen sich auch die Hydroxyde von $\mathrm{Mg}$, $\mathrm{Zn}, \mathrm{Cd}, \mathrm{Hg}, \mathrm{Mn}, \mathrm{Ni}, \mathrm{Co}, \mathrm{Cu}$, also die Hydroxyde von der allgemeinen Formel $\mathrm{M}(\mathrm{OH})_{2}$. Die Lösungen, welche Hydroxyde von der allgemeinen Formel $\mathrm{M}(\mathrm{OH})_{8}$ und $\mathrm{M}(\mathrm{OH})_{2}$ enthalten, sind alle rotbraun gefärbt. Um die Lösungen zu erhalten, fügt man dem Chlorid unter stetem Umrühren - um den Prozeb zu begünstigen … langsam nach und nach gelatinöses, frisch hergestelltes Eisenhydroxyd hinzu. Wenn die Lösung eine rotbraune Farbe aufweist, unterläßt man das weitere Hinzufügen von Hydroxyd, da sonst eine Ausfällung der ganzen Lősung stattfinden kann. Fs ist mir gelungen, in derselben Eisenchloridlösung alle erwähnten 